\title{
Paying the Pipeliner: Academic Investment in Early Stage Drug Discovery
}

\author{
Jeffrey Aubé \\ Professor, Medicinal Chemistry \\ The University of Kansas
}

$\mathrm{T}$ he development and distribution of new drugs requires the collusion of an extraordinarily broad spectrum of professionals ranging from pharmacologists to nurses to marketing specialists. Because of the intellectual and technical challenges associated with this endeavor and also the potential rewards that accompany success in this field, interest in drug discovery research in non-traditional venuesthat is, outside of the pharmaceutical industry-has burgeoned in recent years. Scientists and physicians within the academy, however, have played roles since the invention of the field. John Langley (1852-1925), co-inventor of the receptor theory of drug action still used today, was a professor at Cambridge, and clinical trials

of advanced drug candidates are routinely carried out in academic hospitals worldwide. What is new is that universities, hospitals, and state or local governments interested in economic development have greatly expanded their activities relevant to early stage drug discovery or preclinical research as part of larger initiatives in bioscience.

In this paper I consider some of the scientific and economic aspects of this trend. The conclusion will be that expanded involvement of basic scientists in preclinical research is a welcome and exciting trend from a scientific perspective, but that those who invest time and money in these endeavors should ponder carefully their expectations of return on investments.

\section{The drug discovery pipeline}

The road from laboratory to bedside is fraught with difficulty. A typical drug discovery program takes 10 to 12 years between the initial synthesis of a new prospective drug molecule and its entry into the marketplace. Of course, every campaign is different, and there are some differences between the development of traditional, oral medications and other classes of biological agents such as vaccines. In any event, a time frame of 12 years between initial synthesis and patenting must be considered in the context of the 20-year limit given to drug patents. Thus, a new drug has only about eight years of exclusive marketing to accrue profits to offset the cost of developing the agent. It is well known that post-patent competition by generic drug firms greatly decreases the sales of name-brand drugs. For example, Eli Lilly's Prozac ${ }^{\circledR}$ had $\$ 2.6$ billion of exclusive sales in 2000; after coming off patent, Lilly's market share of fluoxetine 
(the generic name of Prozac $®$ ) dropped to where the company grossed $\$ 0.73$ billion for the same drug in 2003-a drop of over $70 \%$. In addition, the industry depends on its profitable drugs to offset the accumulated costs of all of their unsuccessful drug campaigns, which make up the majority of their efforts.

The odds against a given chemical entity making it into the clinic and from there onto the general market are staggering. It has been estimated that anywhere from 10,000 to 100,000 discrete new chemicals are synthesized en route to successfully introducing a new medicinal agent. Currently, the entire pharmaceutical industry, which employs thousands, is introducing about 30 new drugs into the market per year in the United States. The odds that a given medicinal chemist will produce a new drug are accordingly very low; an anecdotal survey conducted by the author indicates that this fraction is less than $1 \%$.

The costs of developing a drug discovery reflect these odds. A recent analysis of available data suggested that the average cost for a new drug could range anywhere from $\$ 882$ million to $\$ 1.65$ billion dollars.1 The escalating cost of the drug discovery process is only one piece of the ongoing public discourse on the escalating cost of the drug discovery process is only one piece of the ongoing public discourse on the financing of the pharmaceutical industry. Other elements of this conversation, in particular the topic of drug pricing, are beyond the scope of this paper. It must be recognized, however, that given our

\footnotetext{
${ }^{1}$ http://biag.org/BIAG/art3.htm
}

current privatized model of pharmaceutical development, the profits earned from each successful drug are needed to pay for projects that fall short of the market. Given this reality, drug companies typically choose their targets based on market considerations in addition to biomedical need. One ramification of this is big pharma's emphasis on drugs for conditions, often chronic, that afflict large numbers of America's increasingly gray population, such as for cholesterol control, diabetes, or Alzheimer's disease.

What costs so much? The drug discovery process can be considered in two parts: pre-clinical development and clinical trials. This process starts with the identification of both a global goal, such as the treatment of a particular disease, and a specific biological hypothesis, usually involving a target such as an enzyme or particular cellular process. For drug discovery to commence in earnest a lead compound must be found and an appropriate set of biological experiments mounted. This begins an iterative process in which waves of compounds are subjected to different biological assays and the information gleaned is used by chemists to zero in on better compounds. Successive generations of molecules are examined until the team is able to identify 1 to 3 compounds that have the potential to be a drug.

As noted before, many thousands of compounds may be tested before a team is ready to undertake clinical trials, which is the first point at which compounds are dosed in humans. This is because a successful drug must satisfy a broad range of criteria, of which the 
ability to act at the originally hypothesized target is only one. These include the study of how a particular compound is metabolized by the body, which is critical in determining whether the blood levels will be high enough for the drug to be efficacious while avoiding toxic levels. Safety, of course, is of paramount concern and is addressed in preclinical research by some in vitro (test tube) experiments but mostly through studies carried out in animals. Although computers play an increasingly important role in pharmaceutical research, it is still beyond the abilities of pharmaceutical scientists to design a drug based wholly on theoretical methods.

A preclinical discovery program will result in the identification of one main candidate compound along with several back-up agents that could be brought forward should problems arise with the candidate. Clinical trials are carried out in phases. The first phase is relatively small and involves the dosing of healthy volunteers with the drug with an eye toward determining the safety of the drug and establishing the appropriate dose for humans. Phase II trials mark the first time a drug is tested for efficacy in its appropriate patient population. This process continues into Phase III, which has the same general purpose as Phase II, but is much larger in scope and will typically involve many different testing sites and hundreds of patients. Even after a drug has made it onto the market, the medical community continues to monitor patients using the drug. It may take exposure to many thousands of patients to discover rare side effects of drugs, for example. Conversely, additional benefi-cial uses of drugs are sometimes found after initial marketing for the primary indication.

To the chagrin of discovery scientists, it sometimes seems as though nothing is too small to knock a potential drug out of consideration or end a program entirely. Some setbacks are obvious, such as unacceptable toxicity. Others are technical issues that nonetheless serve as deal-breakers, like the inability to formulate the drug for oral administration. In some cases, problems with manufacturing or procuring the drug can arise, particularly in the case of complex chemicals derived from nature. A high-profile example of this concerned the anticancer agent Taxol®, which was nearly scuttled due to since-resolved concerns with harvesting the drug from the natural habitat of the Pacific spotted owl.

Despite the cursory nature of this overview, it should be clear that drug discovery requires early stage contributions from a remarkable range of professionals. The list includes, but is not limited to, molecular biologists, biochemists, organic chemists, analytical chemists, pharmacologists, metabolism experts, and physiologists. The participation of physicians and other medical professionals increases as one enters clinical stages, but there is nothing to prevent participation of any of the above professionals throughout the range of drug discovery activities.

\section{Academic involvement in early stage drug discovery: risks and opportunities}

It should be clear from the above list of disciplines that numerous oppor-tunities exist for the involvement of academics in 
drug discovery; the role of currently highest profile is that of academic hospitals in clinical trials. In contrast, the number of drugs that were originally discovered in academia is very small. Ironically, this stands in strong contrast to the commonly held opinion that drugs usually are discovered by academic scientists using public funding for their projects.

Of course, examples of marketed drugs that were originally discovered in academia do exist (there are enough that a full list is beyond our scope; only a few examples will be cited for illustration). An early landmark was the invention of important anticoagulants and other health-related processes at the University of Wisconsin in the early part of the 20th century. This work eventually led to the formation of the Wisconsin Alumni Research Foundation (WARF), which supports UW research to this day. University faculty members have been connected with drug discovery in other ways as well, often leading double lives as academics and entrepreneurs. One common model is for the faculty member to identify a promising line of research in her or his academic work and then to found a small company that is charged with patenting and commercializing the invention. Some academic entrepreneurs have even become famous outside of academic circles, a classic example in chemistry being Carl Djerassi, who has been widely celebrated for his role in the development of the birth control pill.

Several recent cases illustrate how financial participation in a successful drug launch can have a transforming influence at a university able to obtain it. Three cases involving medicinal or organic chemistry bear mention. In 1991, Professor Robert Holton of the University of South Florida was granted a patent for the synthetic process that ultimately solved the Taxol® supply problem, generating a very substantial income stream for FSU and in the process making Professor Holton a millionaire many times over. Two other cases involved anti-HIV drugs and required legal action by the respective universities to receive royalty incomes from the inventions. In one case, the University of Minnesota ultimately settled with GlaxoSmithKline in an amount estimated to exceed $\$ 300$ million to be split between the university and faculty inventors. A few years later, Emory University and its researchers reached a deal with Gilead Sciences and Royalty Pharma to receive $\$ 525$ million for the sale of its rights to emtricitabine.

Needless to say, the sheer size of these settlements is enough to put stars in the eyes of potential faculty inventors and university administrators alike. Perhaps spurred on by stories like these, both universities and their sponsors - be they endowments or state governments -have tuned into the economic growth opportunities offered by health science research and development in both the academic and private sectors. In Kansas, the Kansas Economic Growth Act, approved in 2004, designated funds of more than $\$ 500$ million over 5-10 years to be used for bioscience initiatives. Although most of the action in start-up drug discovery takes place in clustered areas, such as the California Bay Area, San Diego, Boston, and the Research Triangle Park in North Carolina, bioscience businesses are springing up in 
many non-traditional sites. Such companies need a well-educated workforce that, although recruited nationally or internationally, typically prefers life in a big-city or college-town environment. Depending on the level of capitalization, access to certain infrastructure is often necessary as well, ranging from high-dollar instrumentation to technical libraries. In those cases where a company is based on technology that had its genesis in a university laboratory, proximity to the inventor (who typically retains an academic appointment but has financial interest and a founder's title in the start-up) may be desirable. In return, such companies add to the tax base in their hometowns, provide well-paying jobs for their employees, and can burnish the overall business environment of their communities.

It is clear that major research universities offer many of these amenities through their own academic/research infrastructure, as a ready source of technically qualified personnel, and by the transforming influence they often have in their home communities. Furthermore, many are waking up to the realization of the value, in hard dollars and cents, that academic discoveries may have in the "real world". The result is that more major universities than ever are seeking to promote drug discovery research, of all types and at all levels, within their walls. In some cases, the goal is clearly to replicate many of the elements needed to bring a molecule from the laboratory to the bedside, all within the academic environment.
A final element is the increasingly difficult funding climate across all areas of science. Always cyclical, as of this writing funding levels are at their lowest levels in many years. Despite the muchvaunted recent doubling of the budget of the National Institutes of Health, funding rates are currently limited to the top 7$12 \%$ of proposals considered at a given funding cycle (overall rates are slightly higher because they take multiple submissions of a given project into account). For many academic labs, successfully obtaining this funding is a major determinant whether they will thrive and in some cases even survive. Individual investigators and, by extension, the institutions that employ them, have increasingly looked to drug discovery-related activities as a way of getting a competitive edge in the competition for these critical funding sources.

What are the risks and possible rewards of these endeavors? To what extent is drug discovery work appropriate for faculty, staff, and students in institutions of higher learning? The central argument of this paper is that such work is both appropriate and healthy for universities that have that the will and the way, but that those undertaking it should be aware of the limitations of this work and have a clear view of what they hope to accomplish along the way. I will briefly consider various ramifications of early stage drug discovery in academia in the remainder of this section. All opinions expressed are mine and do not necessarily reflect those of my employer or my colleagues. 
Drug discovery research is an exciting field that is appropriate for many traditional academic disciplinesand some new ones. For many scientists, drug discovery is "where it's at". As basic sciences such as chemistry, physiology, and biology have successfully tackled many of their classical challenges, the move toward interdisciplinary bound-aries has increased. "Research at the interface between field $X$ and field $Y^{\prime \prime}$ (insert specifics as needed) has become a cliché in leading journals and graduate school recruiting brochures alike. In addition, other fields like pharmacology and such hybrid disciplines as medicinal chemistry and chemical biology are of obvious intrinsic relevance to various stages of the drug development process. This excitement extends to NIH funding panels. While $\mathrm{NIH}$ reviewers have always understood that basic scientific advances are needed to fuel future advances in human health, they are nonetheless increasingly interested in funding projects with a shorter term for payoff.

Doing cutting edge research serves traditional academic values. Besides the obvious rewards to the faculty researcher, which include publications and greater competitiveness for research funding, strong programs in the field provide an excellent education for graduate student and postdoctoral colleagues, who in turn become more valuable on the job market for either academia or industry. Importantly, such benefits accrue whether or not commercialized products lead to income generated through royalties or licensing.
Researchers who work on the drug discovery pipeline have the responsibility to conduct their work with high ethical standards. A common criticism levied is that researchers seeking to work in drug discovery are seeking to do "trendy" research or are in the pockets of the pharmaceutical industry. In my opinion, this viewpoint is uninformed. First, as argued above, the move toward much "applied" work in drug discovery is mostly a product of the natural evolution of the basic academic disciplines. Secondly, relatively few researchers receive funding from pharmaceutical companies for their work. Those who do enter into collaborative research agreements are generally further down the pipeline, closer to commercial development, and are working to exploit early stage discoveries already made in their laboratories. In still other cases, drug companies sponsor awards or fellowships that are given to highprofile, usually young, investigators. Most of these "beauty contest" grants are designed to generate favorable publicity for the company instead of seeking to prejudice the work in the professorial lab. Although one must always be vigilant about the possibility for misuse, this writer is not personally aware of any case in which a faculty member so honored has been implicated in any kind of tainted study.

There are legitimate concerns that arise when academic researchers seek to adopt a more business-oriented model for their efforts. It only takes a cursory glance at the newspapers to get a drift of the temptations to fudge data in the drug development business and there is no 
reason to assume that academics would be immune to the same pressures as their industrial colleagues. It is clearly in the best interest of researchers, universities, and the public for all drug discovery workers - in industry and academia - to adopt the most rigorous scientific standards in their work and nothing less should be tolerated.

In the meantime, it would be appropriate to keep in mind that although drug research is expensive and has the opportunity to bring in valuable grants and possibly other kinds of income, healthy and successful universities benefit from diverse academic discourse across a the whole range of human endeavors. Fine arts, humanities, social sciences, business, and other academic disciplines may often cost less but they are certainly not less important. In the end, there is more to scholarship than money, and scholars should not be judged on the size -or even existence - of their research accounts.

Everybody-government, academia, and the public-should carefully consider their expectations for return on investment in drug discovery efforts. For those working on drug discovery research, perhaps it should go without saying that the ultimate reward is actually succeeding in the overall task and bringing a drug to market. And it does happen: witness the stories above for drugs tied to work at Wisconsin, Minnesota, and Emory. In 2006, Professor E. C. Taylor of Princeton University was honored as a "hero of chemistry" by the American Chemical Society for his role in the development of the Lilly anti-mesothelioma agent
Alimta ${ }^{\circledR}$. Professor Valentino Stella of the University of Kansas is an inventor of fosphenytoin, an injectable anti-epilepsy agent. Such cases can be win-win-win, with a new drug made available to a patient population that needs it and financial rewards accrued to both inventors and their employers. Universities that are beneficiaries of drug royalties can use them to establish ambitious new programs in drug discovery or other fields and, in times of difficult funding for research in particular and higher education in general, such windfalls provide remarkable opportunities for future growth. The WARF program at Wisconsin is an impressive model for the long-term possibilities of drug discovery successes.

This kind of success, however, is still relatively rare, and the road from idea to a new drug is harder than ever. Indeed, all of the cases mentioned in this paper involved an established drug company en route to market. More tellingly, several of them also involved lawsuits, generally settled out of court, to sort out which contributions were made by the academic researchers and what sort of reward was ultimately warranted. To go the distance in the "big pharma" model takes both determination and luck to get one of the ca. 30 drugs approved each year. Overall, it seems unrealistic for universities, their endowments, or state governments to invest in drug discovery research with the expectation that a billion-dollar drug is the natural outcome of these efforts.

Science and medicine typically advance incrementally and alternative models recognize this. Even unsuccessful 
drug discovery campaigns provide useful knowledge in basic science and can suggest more fruitful pathways for future endeavors. Consider the way molecules move through the pipeline. The "lead compound" described in a previous section is an essential starting point for the pathway to drug discovery. Far from being a commodity item, useful leads that can be advanced to clinical candidates are very difficult to find, requiring extensive "needle in the haystack" enterprises such as highthroughput screening or natural product prospecting just to get started. Accordingly, promising leads are highvalue items from which substantial licensing income can derive. For smaller biotech companies, the discovery of such a lead that is then peddled to major pharma constitutes a significant business success; sometimes the small company is bought outright. It is reasonable to expect that leads discovered in universities, which have access to modern biology and chemistry expertise will continue to be attractive to the pharmaceutical industry that is interested in fresh opportunities.

One can maximize the possibilities of finding drug candidates by looking in unusual places or through renovation of already-existing agents. As noted above, the sheer expense of drug discovery has placed most large drug companies in the position of concentrating on largemarket patient populations. Accordingly, a significant unmet need exists for many so-called "orphan diseases". One could argue that work in this area not only represents an opportunity for smaller pharma and academic efforts-both of whom would certainly benefit from the marketing of an agent that might earn "only" 100 millions dollars a year-but that alternative drug prospectors have a moral obligation to serve people who are suffering from non-mainstream conditions. National agencies have also recognized this need. Perversely, one area that has been all but abandoned by mainstream pharma is the discovery of new antibiotics. Despite the threat of emerging resistance and gaps in current treatment capabilities, antibiotic research in big pharma has diminished because it has been deemed financially unsoundthus creating another opportunity for academia to fill a major need.

Despite the focus of this discussion on the molecular aspects of drug discovery per se, other disciplines and skills are equally essential and provide prospects for research in fields ranging from biology to information science. Products and processes invented in such areas have similar potential for scientific advancement and possible commercialization.

All of the above requires a soberminded approach to the issue of intellectual property. Although relatively straightforward for efforts carried out totally within university walls, the involvement of external collaborators can complicate IP matters rapidly. There are numerous complaints from both sides of the fence, chiefly pertaining to unrealistic expectations on the part of academics regarding the value on unverified leads and concerns that industrial expectations for secrecy will interfere with essential elements of the academic/public mission of universities. Some thought as to how these issues will be considered is essential at the outset of any 
collaborative effort between the academic and private sectors.

New funding mechanisms encourage team-based research in academia. Given the sheer diversity of expertise that must come together to effect real drug discovery in academia, modern biomedical research is increasingly looking like the "big science" model that has been prevalent in some areas of physics for quite some time. Real progress in drug development requires not only an array of expertise but also their intersection. This has been increasingly true with the prevalence of new technology that has accelerated drug discovery, but which requires specialized technical knowledge and sophisticated equipment. Two technologies that stand together at the beginning of most drug discovery efforts are high-throughput screening and highthroughput synthesis; both of these seek to accelerate drug discovery by carrying out biological examination of new druglike substances with the aid of automation. Increasingly, collections of chemical compounds called libraries are synthesized and examined in parallel, helping to decrease the time needed for examining the many thousands of compounds needed for a given drug discovery campaign. Both techniques are ubiquitous in industry but have been more slowly accepted in academia due to cost as well as lack of familiarity. Greater penetration of these techniques into academia would result in an additional benefit, as industry also takes advantage of traditional academic innovation as revealed through the scientific literature.

This issue has been recognized by the National Institutes of Health through parts of the NIH Roadmap for Discovery. This initiative, announced by NIH director Elias Zerhouni in October 2003, is a sprawling enterprise that seeks to coordinate biomedical research to better handle modern, complex problems (the motto is "accelerating medical discovery to improve health"). One major element of the Roadmap is devoted to molecular libraries and screening and has worked to encourage centers in both screening and synthesis throughout the U.S. (Similar initiatives have been mounted in Canada.) Such centers provide the academic community with important resources that would otherwise be unavailable through single-investigator research grants. In addition, the NIH is putting together a model for nationwide collaboration between chemists and biochemists through its screening program, the hope being that it will prove possible to greatly enhance the number of chemical tools available for biological research. It is important to recognize that drug discovery per se is not the stated objective of the NIH in this initiative. Rather, the Roadmap seeks to enable projects in basic biological and pharmacological science and to provide new techniques and tools for biologists and chemists. The differences between this kind of basic biology and drug discovery lie largely in how the knowledge gleaned is applied. Thus, there is no cross-purpose in doing Roadmap biology or in seeking to develop new drugs; excellent science in the former activity leads naturally into the latter.

Not surprisingly, center programs, which also operate by peer-reviewed funding mechanisms, have drawn 
criticism for the perceived budgetary harm they do to single-investigator programs. And although center grants are both prestigious and typically bring substantial support, they also make demands on universities that receive them. Specifically, many call for the establishment of core laboratories that do specialized research and in some cases provide scientific services to a larger community. To name one example, a core laboratory in high-throughput synthesis will require sophisticated and expensive robotics (which are considerably more costly than beakers and flasks!).2 In turn, this equipment requires laboratory space, which is always at a premium at academic institutions. Most significantly, the maintenance and operation of core laboratories often requires full-time staff, especially when a service activity is expected. Thus, although large center projects may be attractive from the perspective of bolstering the bottom line, the investment in time and infrastructure that they require mean that it only makes sense to take part such programs if they positively contribute to the long-term interest of the organization and its investigators. In other words, there has to be an exit strategy: why invest years of one's finite active research life and considerable financial costs to build up any given research infrastructure if there is no plan for its continuance beyond the 5-10 years of support that is standard for federal agencies? Although it never makes sense to undertake any line of research only because "that's where the

\footnotetext{
2 The author discloses that he is the director of such a center at The University of Kansas funded by the NIH (www.cmld.ku.edu).
}

money is," such investments can pay off when there is a confluence between an individual's particular research interests and programmatic opportunities. Given the long term requirements of the great majority of drug discovery campaigns, success requires both patience and longterm institutional commitment.

A number of institutions are taking exactly this approach, sometimes dipping into endowments or courting donors to fund such programs on their own terms. This has been most commonly associated with medical schools (Vanderbilt's, Sloan-Kettering's and St. Jude's Children's Research Hospital's programs stand out). There is also the "nothing succeeds like success" approach, in which those programs that have already established an income stream from drug royalties have committed them to long-term activities in biomedical research. None of these models is open to more than a handful of universities, meaning that a combination of grant success (which is to a degree self-perpetuating) and shrewd, focused investment of overhead return or endowment will be the most common approach. In the event that an actual drug launch becomes a possibility, private sector involvement is eventually necessary with the concomitant issue of intellectual property.

The Bottom Line: There has to be a long-term commitment to drug discovery that makes sense in the overall vision of the university. Drug discovery is an exciting, contemporary activity that attracts the attention of an increasing number of academic scientists. All who wish to undertake drug discovery in academia, however, should carefully 
consider their expectations and both of which are available to a great commitment. The chance that any given range of disciplines and academic investigator - in big pharma, biotech, or temperaments. Bringing drug discovery academia-will bring a new drug to into the academic setting provides an market is small. However, much is to be opportunity to contribute to human gained by engaging in basic science health in areas that are underappreciated related to human health. The best in the global pharmaceutical industry reasons to engage in drug discovery are and to train a new generation of scholars scientific excitement and a desire to do who are committed to contributing to the work that is relevant to human health - betterment of humanity. 